\title{
Conceptual Issues for Noncommutative Gravity on Algebras and Finite Sets
}

\author{
Shahn Majid \\ School of Mathematical Sciences, Queen Mary and Westfield College \\ University of London, Mile End Rd, London E1 4NS円
}

\begin{abstract}
We discuss some of the issues to be addressed in arriving at a definitive noncommutative Riemannian geometry that generalises conventional geometry both to the quantum domain and to the discrete domain. This also provides an introduction to our 1997 formulation based on quantum group frame bundles. We outline now the local formulae with general differential calculus both on the base 'quantum manifold' and on the structure group Gauge transforms with nonuniversal calculi, Dirac operator, Levi-Civita condition, Ricci tensor and other topics are also covered. As an application we outline an intrinsic or relative theory of quantum measurement and propose it as a possible framework to explore the link between gravity in quantum systems and entropy.
\end{abstract}

\section{Introduction}

There has been a lot of interest in recent years in a theory of 'Riemannian geometry' and gravity applicable to general possibly noncommutative, possibly discrete algebras. The motivations are obvious enough and have been featured in many a research proposal in the last several years. In this note I want to discuss some of the issues in arriving at the proper formulation as well as to provide an introduction to my own solution to this problem some years ago, and which I presented in detail at the Euroconference. Probably the biggest problem with this formulation is that it is technically too hard for most readers; I will try to address some of that here. I also want to put in writing the announcements made in my lectures of some of my results, which will appear in the long paper [1] in preparation.

We start with some motivation for such a theory, which in my case (for about 15 years) has always been quantum gravity or Planck scale physics. The first paper that I am aware of in which actual noncommutative geometries based on quantum group methods were proposed as a model of Planck scale physics was [2]. We put forward here (in 1987) the view that:

A noncommutative noncocommutative Hopf algebra should be viewed as a toy model of a system in which both quantum effects (the noncommutativity) and gravitational curvature effects (the noncocommutativity) are unified.

Many years later, with a theory of quantum Riemannian geometry, we can now actually measure the curvature in a (possibly) quantum system, for example relating it to the noncocommutativity in the case of a Hopf algebra or quantum group, and thereby make this idea precise. Many inspired works were born from semantic confusions and here I want to note for example that if one views one of Drinfeld's quasitriangular Hopf algebras $U_{q}(\mathfrak{g})$ this way (as a 'coordinate algebra', which is not the usual way) then one can expect the following identity

$$
R_{\text {Riemann }} \sim \mathcal{R}_{\text {Drinfeld }}
$$

where the latter is the quasitriangular structure or universal R-matrix that controls the noncocommutativity. The theory to be described below has the power to make this precise.

\footnotetext{
${ }^{1}$ Reader and Royal Society University Research Fellow
} 
More physically, the paper [2] introduced some concrete ideas for the Planck scale relating to a duality between quantum theory and geometry which could be viewed as a modern version of Mach's principle, and such ideas could be explored now in more detail. One should certainly like to have more realistic and relativistic models with similar phenomena. Also in general terms, there are many indications of a profound relationship between gravity, statistical mechanics and the 'problem of time'. The tools of noncommutative Riemannian geometry would provide the power to resolve those relationships in detail. We would be able to deal with algebras which are actual quantum systems, compute entropy of states etc, and at the same time compute their geometrical structure, curvature etc, without classical limits, and hence sort out these issues algebraically (I would guess that they relate to some form of the above -mentioned duality). Again, this has been explored in the very simple case of Hopf algebras, at least a little, via 'quantum random walks' on them[3] but before the tools of Riemannian geometry were available.

Another impetus is that string theorists are finally beginning to accept this view (which was not true 10 years ago for example) although the noncommutative tori found there (at the algebraic level at which physicists tend to work) are too trivial to be good candidates for quantum spacetime, being the usual Heisenberg or Weyl algebra in one form or another.

In a different direction, one can use noncommutative geometry methods to regularise even ordinary quantum field theories on flat space. The first result of this kind I believe appeared in 1990 in [4] where it was proposed to consider the enveloping algebra $U_{q}\left(b_{+}\right)$'up side down' as a noncommutative spacetime and shown that this leads to better convergence of some integrals. In the limit $q=1$ our proposal was for $[x, t]=x$ to be spacetime. Recently such noncommutative 'kappa-Minkowski' spacetimes have been studied using the integral on this algebra in [4] and ensuing Fourier transform to make the first mathematically meaningful predictions for gamma ray propagation that could be tested in principle by cosmological data[5]. Also many years later, regularisation by q-deformation is finally becoming a reality for viable quantum field theories, as shown by Robert Oeckl's talk at this conference; see his seminal paper [6].

The improved properties for regularisation can be traced to finite differences rather than usual differentiation. The natural emergence of these in noncommutative geometry obviously makes any successful Riemannian geometry of that type also a natural and less ad-hoc replacement for lattice and other discrete modeling methods for quantum gravity. One can either work with a finite-difference calculus on classical (not lattice) spacetime by choosing an appropriate noncommutative differential calculus, or one can go further and make the space or spacetime itself discrete or finite. This is another obvious application of a successful noncommutative Riemannian geometry.

Also, Connes some years ago gave an approach to the standard model of elementary particle physics in which conventional internal gauge symmetries are recovered (along with the Higgs potential) in a much more elegant manner than usually presented, by extending spacetime by a finite-dimensional noncommutative algebra or 'discrete' part. The problem with this approach is that some of the most interesting physical data relating to masses in the standard model is encoded in the 'Dirac operator' on the finite algebra, but this can be practically any self-adjoint operator. If we had a true quantum Riemannian geometry that included discrete or finite systems then we would know what was the natural or canonical Dirac operator in a given context from a geometrical point of view and thereby obtain direct physical predictions about the standard model. So the same technology would have a second quite different application to physics. One can go further and relate it to quantum gravity as well through Connes more recent 'spectral action principle'.

If this list of motivations is not enough, I want to present a final one. This is that one can gain geometric tools for ordinary quantum mechanics and address fundamental interpretational problems there. For what is the correspondence principle other than the idea that certain operators in the quantum theory like $x, p$ behave similarly enough to their classical counterparts that we correspond them? Noncommutative geometry for the first time offers a way to turn that very woolly idea into something precise, i.e. to extend the correspondence principle itself into something rather more sophisticated in which we say that a given quantum algebra has this or that quantum geometric structure and therefore identify correctly the pieces for its classical geometric limit. Actually this is not something just for philosophers of quantum mechanics; if we are going to achieve 
and understand quantum gravity we will probably need to fully understand the measurement process in a more intrinsic manner than 'somebody outside' making a measurement. This is somehow tied up with the entropy questions we began the discussion with.

Let us now summarise some of the requirements stemming from the above motivations. I would identify 4 key demands for noncommutative Riemannian geometry.

1. The theory should have full conceptual continuity with conventional Riemannian geometry so that this is included as a special case (along with finite geometry and noncommutative algebras as other specialisations).

2. There should be either a global picture (for nontrivial topology) of the whole theory or local gauge transformations from which a global picture could be patched.

3. The theory should be powerful enough to include a wide variety of genuine examples covering a full range of objects that people would want to consider 'geometrically' and with similar or more degree of 'flabbiness' as usual geometry.

4. The theory should probably in principle include spinors since these are needed for matter fields.

To give an example of a theory failing 2., supermanifolds are basically ordinary manifolds with a 'Grassmann' part glued on; the latter is always flat and not really capable of its own topological structure. For an example of an approach failing 3., the 'idea' that vector fields should be derivations is obvious (and makes sense for any noncommutative algebra) but works only for very special matrix type algebras; in many other cases, (e.g. for quantum groups), the natural partial derivatives are not derivations at all, and indeed essentially none exist. This problem may also occur in Connes' spectral triple approach where, without a full range of noncommutative examples, one cannot be sure about the applicability of the axioms even if they are correct in the commutative case and mathematically elegant. That is why I want to emphasise 3 .

Here at this conference several interesting talks were related to these topics. Sorace made some steps towards Dirac operators on Euclidean quantum groups, while Castellani made an attempt at a theory of gravity on finite groups. None of the works, even in the finite or discrete case, however, fit our primary demand of conceptual containment and continuity with classical geometry, either missing key definitions such as compatibility between the so-called 'spin connection' and the metric, or using ad-hoc definitions heavily dependent on a lattice or group structure on the spacetime, or limited to some form of universal calculus much bigger than classical. Also on point 4. it should also be said that essentially all attempts at noncommutative Riemannian geometry theories other than the frame bundle approach introduced in [7] have been based on positing the covariant derivative $\nabla$ directly via certain axioms, i.e. a theory of linear connections. Such an approach can never interact properly with matter fields, particularly spinors, since these need at their base a principal bundle of some sort to which vector bundles such as the spin bundle are associated. The only way that has been found to circumvent that is Connes's approach to jump over covariant derivatives altogether and posit the 'Dirac operator' directly as the basic object, as mentioned above. However, in this approach it is hard to pick out connections or much else of the infrastructure of differential geometry at all, other than in the commutative case, i.e. one cannot test our primary 'continuity' requirement around the commutative case. Connes' axioms may in fact have to be significantly modified to reach the standard q-deformation examples, for instance.

I would like to stick my neck out and claim that our 1997 frame bundle approach to Riemannian geometry in [7] on the other hand does already more or less meet all four criteria at least in principle. The paper itself focussed on two extremes, checking continuity with the classical case and at the other extreme the universal differential calculus, but it also indicated the theory for general calculi using the principal bundle theory for general differential calculi already known by then in [8], i.e. this was a matter of presentation and not a fundamental or conceptual problem with the theory. Basically, the examples had not been worked out for nonuniversal calculi to justify a full presentation, an oversight that will be filled in the forthcoming paper [1] with examples for all standard quantum groups and their standard calculi, as announced here in Torino. 
It should also be clear in all this the role of quantum groups themselves: I would view them as nothing but a good playground of 'naturally arising' and evidently geometrical objects, just as Lie groups provided the perfect playground for the development of modern classical differential geometry in the late 19th and early 20th century. Particularly, q-deformed examples, because of their parameter, allow us to test the continuity of our noncommutative Riemannian geometry on an 'open set of models' near the commutative point. This goes much beyond simply having the right answer in the commutative case. However, any theory of 'Riemannian geometry' that works only for quantum groups, or only for finite sets, etc., will not meet our key demand and will most likely be viewed as ad-hoc when the full theory is known. This excludes all sorts of rigid theories based on integrable systems etc; they should be examples of some general theory but are not that theory.

In fact, some examples that one would wish to include already force one to generalise away from a quantum group as 'gauge group' to a general algebra (or rather, coalgebra in our dual language) in its role. It turns out that most of what we want to do can be done (with a lot more care) at that much more general level[9] but along exactly the same lines as in [7]. This step is certainly important to meet our demand 3. and we will say more about it in the last section, but at the moment only the special case of a quantum group framing (which could of course be a classical or finite group if one wants) has been more or less fully worked out with general differential calculus.

\section{Size matters for differential calculi}

We set the scene with a brief discussion of the choice of differential calculus, which in common concensus means a choice of exterior algebra $\Omega(M)$ for our 'coordinate' algebra $M$. $M$ could be the functions on a finite group, a matrix algebra, a quantum group etc. As in conventional geometry one can focus on each degree of differential forms one at a time, i.e. first define $\Omega^{1}(M)$ and let the higher order be unspecified until one needs them. More formally, one can say that the higher order just have the relations among higher order forms implied by Leibniz and $\mathrm{d}^{2}=0$ from what was assumed at degree 1 . This is the 'maximal prolongation'. On the other hand, that is not at all what would give the right answer in the classical case. There will typically be an infinite exterior algebra with forms of all degrees and no top form, etc., because sufficiently many relations at higher degree are not demanded by Leibniz and $\mathrm{d}^{2}=0$ alone.

At the moment there are only three ways to specify a reasonable calculus to all orders of differentials. In either case one starts with the universal exterior algebra $\Omega M$ associated canonically to any algebra - this has very little structure at all and is far far too big to be classical. One then specifies the quotients at each degree by some scheme to get down to a calculus of 'reasonable' size at each order. For example, at degree one, the universal calculus $\Omega^{1} M$ is the subspace of $M \otimes M$ given by the kernel of the product map. Its universal d is

$$
\mathrm{d} m=1 \otimes m-m \otimes 1, \quad \mathrm{~d}: M \rightarrow \Omega^{1} M .
$$

It is a bimodule so we can multiply forms by 'functions' $M$ from the left or right. Any other choice $\Omega^{1}(M)$ is specified by quotienting, i.e. by setting to zero some subbimodule $N_{M} \subset \Omega^{1} M$. In almost all cases, e.g even for finite sets, the quotients will remain bimodules with different left and right multiplications, i.e. 'quantum' in the sense that forms and functions do not commute. In this sense the commutative calculus that we are familiar with classically is very artificial and atypical.

The problem is that one needs a scheme to specify this quotienting to all orders or at least to the orders needed for physics (which for gauge theory means mainly to order 2 or 3 ). One scheme is to mimic classical ideas. For example one can have a 'discrete geometry' (where $M$ is the algebra of functions on a discrete set) by starting with some existing manifold or more generally a sigma-algebra and use the intersection structure of a good open cover to specify a calculus on the indexing set of the cover, see for example [10] (which differed from other similar proposals). This would give an example of a discrete version (using noncommutative geometry for the differentials) of your favorite classical manifold.

Another scheme is to suppose that the space has a group structure, i.e. $M$ is a quantum group (or classical group coordinate algebra). The coproduct $\Delta: M \rightarrow M \otimes M$ expresses the group structure of course. For 
on a Lie group there is a unique differential structure that is translation covariant from the left and right and it specifies forms to all orders. This was formulated many years ago by Woronowicz 11 who showed in particular that once $\Omega^{1}(M)$ was fixed in a bicovariant manner then there was a natural $\Omega(M)$ of the right kind of size (reducing correctly in the Lie group case). The basic idea is to set to zero those products of forms that would be invariant under a generalised transposition or 'braiding' operator $\Psi$. The braiding is that of the category of representations of the Drinfeld quantum double $D(M)$. Recently by results in [12] [13] and a twisting theorem in [14] the classification problem for the remaining freedom of choice of $\Omega^{1}(M)$ for all main classes of quantum groups was solved. So by now we know the menu of choices of differential calculi in the quantum group case more or less completely.

The third scheme is through Connes spectral triple, i.e. to choose a more or less arbitrary operator $\not D$ that you would like to be able to call Dirac acting on a vector space of the form $M \otimes W$ ( $W$ would be the spinor space) and let it determine the smallest differential calculus on $M$ that would be needed for this to be the case. Most of Connes axioms involving Hilbert spaces etc are not needed for this and one can formulate it all as a purely algebraic construction. It is analysed a little in comparison with the Woronowicz approach in [1]. Basically, consider $\pi: \Omega M \rightarrow \operatorname{End}(M \otimes W)$ defined for example on 1-forms by $\pi(m \otimes n)=m[\not D, n]$ where $m, n$ act by multiplication. Then[15] quotient the universal $\Omega^{1} M$ by $\operatorname{ker} \pi$ to define $\Omega^{1}(M)$. Similarly at higher order we quotient by the differential ideal generated by the kernel of $\pi$. This gives the right answer classically if one really starts with the Dirac operator but all depends on the choice of $\not D$. For a given algebra you may have little or no idea which operator to take to get something finite or resembling classical geometry.

To give an example, for polynomials in one variable over a field $k$ the coirreducible translation invariant calculi have the form

$$
\Omega^{1}=k_{\lambda}[x], \quad \mathrm{d} f(x)=\frac{f(x+\lambda)-f(x)}{\lambda}, \quad f(x) \cdot \alpha(\lambda, x)=f(x+\lambda) \alpha(\lambda, x), \quad \alpha(\lambda, x) \cdot f(x)=\alpha(\lambda, x) f(x)
$$

for functions $f$ and one-forms $\alpha$. Here $k_{\lambda}$ is a field extension of the form $k[\lambda]$ modulo $m(\lambda)=0$ and $m$ is an irreducible monic polynomial. For example, the most important field extension in physics, $\mathbb{R} \subset \mathbb{C}$, can be viewed noncommutative-geometrically with complex functions $\mathbb{C}[x]$ the quantum 1-forms on the algebra of real functions $\mathbb{R}[x][16]$. There is nontrivial quantum DeRahm cohomology in this case.

\section{To gauge or not to gauge; the global approach}

Once you have a differential calculus you can do cohomology, of course. You can also do what I like to call $U(0)$ gauge theory in which we regard a connection or gauge field simply as a 1-form $A$ but transforming as

$$
A^{\gamma}=\gamma^{-1} A \gamma+\gamma^{-1} \mathrm{~d} \gamma
$$

under 'gauge transform' by $\gamma$ an invertible element of $M$. Or a unitary element in a representation of the algebra, say. From a noncommutative geometric point of view in which $M$ is 'functions' this is a trivial gauge theory even though the curvature

$$
F=\mathrm{d} A+A \wedge A
$$

transforms nontrivially by conjugation.

To do gravity on $M$ we need a full nonAbelian gauge theory in which the gauge field has values in a general quantum (or classical) group $H$. Such a theory was introduced in 1992 in [8] and remains the only properly formulated global and nonAbelian gauge theory for a quantum space that I am aware of. More precisely we equip $H$ with a differential calculus $\Omega^{1}(H)$ and consider the space $\Omega_{0}$ of left-invariant 1-forms as the dual of its Lie algebra in some sense, and gauge fields should be maps from $\Omega_{0}$ (i.e. have values in $\Omega_{0}^{*}$ in more conventional terms). We also need an algebra $P$ with its own but compatible differential structure $\Omega^{1}(P)$ which plays the role of the total space of a principal bundle and on which $H$ (co)acts by a coaction $\Delta_{R}$ with fixed subalgebra $M$ and some axiom of 'local triviality'. Remarkably, it is possible to do away with 
all of the usual mess with local trivialisations and local charts (which we will not have for a general algebra) and replace them by an algebraic condition, the exact sequence

$$
0 \rightarrow P \Omega^{1}(M) P \rightarrow \Omega^{1}(P) \stackrel{\text { ver }}{\longrightarrow} P \otimes \Omega_{0} \rightarrow 0 .
$$

Here ver $=(\cdot \otimes \mathrm{id}) \Delta_{R}$ defines for each element of $\Omega_{0}^{*}$ the corresponding vertical vector field $\Omega^{1}(P) \rightarrow P$ along the fiber and the exact sequence says that the forms that they kill should be exactly the ones pulled back from the base. A connection in the bundle is a splitting of $\Omega^{1}(P)$ and can be cast as a connection form

$$
\omega: \Omega_{0} \rightarrow \Omega^{1}(P)
$$

with properties just like in any good book on differential geometry.

So the global theory exists, a nontrivial example, the $q$-monopole, also in [8] showed that it worked, and some generalisations for other example have been made also. But are we happy after 8 years? Not completely because in physics we also think we want to see local gauge transformation formulae like in (3) at least for trivial bundles. And here there is a problem. The problem is that the abstract mathematical and global way works but the local formulae that you know and love have a lot more classical assumptions hidden in them than meet the eye and which have to be appropriately generalised in a smooth manner. Basically, let us consider what should be the nonAbelian analogue of (3) which is a map

$$
A: \Omega_{0} \rightarrow \Omega^{1}(M)
$$

We can consider gauge transform by $\gamma: H \rightarrow M$ in a similar manner but we wont be able to 'conjugate' $\Omega_{0}$ in the correct way. For to make (3) we would want to apply the coproduct twice as a map $\Omega_{0} \rightarrow H \otimes \Omega_{0} \otimes H$, keep the middle output for $A$ and the outer two for $\gamma^{-1}$ and $\gamma$. This is an analogue of conjugating the Lie algebra by the group, but it is not one that works for a Hopf algebra. There is no such map unless we take the universal differential calculus. If we look at what happens classically what we really need is the adjoint action of the group on the Lie algebra. Here we are in luck, there is a well-defined adjoint coaction Ad $: \Omega_{0} \rightarrow \Omega_{0} \otimes H$. We use it, apply $A$ and $\gamma$ and the bimodule structure,

$$
A^{\gamma}=\cdot \circ(A \otimes \gamma) \circ \mathrm{Ad}
$$

The problem is that then the curvature is not invariant as soon as the differential calculus is noncommutative because the $\gamma^{-1}$ buried inside $\gamma \circ \mathrm{Ad}$ is on the wrong side of $A$ in $\Omega^{1}(M)$. This is the fundamental obstruction that has been around for many years now.

Yet there is no problem in the nonAbelian case with the full global picture! After thinking about this for some years the resolution of the problem is as follows [1]. Firstly, we can postpone the problem. After all, what do we want local gauge transformations for anyway except to make sure things patch up to a global picture? Having a global picture we are already free to make bundle automorphisms or gauge transformations as transformations on $P$ - the problem is just the local picture not the concept of gauge transformation itself. It is only in the local picture that formulae like (3) come up. Next, as we elaborate more of the local theory we will see the gauge transformations appearing and eventually get a local formulae like (3), which must exist because global bundle automorphisms exist. To give a rough idea what the eventual local formulae will look like (and to see why the result will be rather too complicated in general just to guess) suppose that you have a single global patch described by a trivial bundle. Such bundles were considered in [8] and one example, dependent already on the choice of trivialisation or 'gauge' is the tensor product bundle $P=M \otimes H$. As already understood there, the new feature in quantum groups gauge theory is that under a gauge transformation $\gamma$ there will be a new trivial bundle with the same vector space $M \otimes H$ but in fact $P$ will no longer be a tensor product but a cocycle cross product

$$
P^{\gamma}=M_{\chi_{\gamma}} \rtimes H .
$$

So the very algebraic form of the bundle changes under a gauge transformation. The cross product and cocycle would all be trivial if $M$ were commutative so you would never leave the class $M \otimes H$ in the classical case; the 
cocycle or 'anomaly' is a purely quantum effect. The new bundle is equivalent but our naive description as a tensor product is not gauge invariant! So there will be a new local picture for $A^{\gamma}$ and a familiar gauge theory but only when we consider the whole class of cocycle trivial bundles $P=M_{\chi} \rtimes H$ stable under such $\gamma$ (with $\chi$ also transforming). There is no problem of principle here at all except that the result is more complicated than we would have liked. With this in mind, we will shortly give all the formulae of quantum Riemannian geometry in 'tensor product gauge' but one should not try to make gauge transformations of them without being prepared to do it in the manner above with a cocycle. Also note that not all trivial bundles are gauge equivalent to a tensor product one (it depends on the vanishing of a nonAbelian cohomology where $\chi$ lives, see $[17$, Ch. 6]), so we will be seeing in this way only the cohomologically trivial sector of the global theory (other sectors never visible classically are also possible).

In effect, our interim solution to the gauge problem is to work globally or work in what I propose to call 'tensor product gauge'. Because the global theory exists it is enough to work (for trivial bundles) in one gauge like this, and consider other algebraic gauges later. It still takes a lot of work 1 to prove that the local formulae indeed lead to a global bundle on $P=M \otimes H$ - we are simply validating them in a different way than by gauge transformation.

And if one really wants gauge transformations at all cost then one can of course use the universal differential calculus on $H$. Then $\Omega_{0} \subset H$ (it is the kernel of the counit) and one can use the coproduct and a projection to define $\Omega_{0} \rightarrow H \otimes \Omega_{0} \otimes H$ as we had first wanted to do. This was also introduced in 1992 in [8] where all usual gauge formulae are given (and this works for general $\Omega^{1}(M)$ ). But such a theory in more usual terms would be allowing the gauge field and curvature to have values in the whole quantum group dual to $H$ as explained in [8]. Such a theory is OK but it violates our principal demand of continuity with classical geometry wherin $A$ and $F$ should have values in some kind of 'Lie algebra' and not in the enveloping algebra to resemble classical geometry. It is only to achieve this that we have to work much harder as explained above.

\section{Quantum Riemannian geometry - global version}

Before passing to tensor product gauge, let us complete the formalism of global quantum Riemannian geometry as basically introduced in [7]. Firstly, in the theory of quantum principal bundles one has the 'associated bundles' $\mathcal{E}^{*}=\operatorname{hom}^{H}(V, P)$ and $\mathcal{E}=(P \otimes V)^{H}$ (the invariant subalgebra of $P \otimes H$ ) defined by any space $V$ in which $H$ (co)acts. Any connection on $P$ then induces covariant derivatives on these,

$$
D: \mathcal{E} \rightarrow \Omega^{1}(M) \underset{M}{\otimes} \mathcal{E}
$$

etc. The new ingredient for Riemannian geometry is a soldering form $\theta: V \rightarrow P \Omega^{1}(M)$ such that the induced map $\mathcal{E} \rightarrow \Omega^{1}(M)$ by applying $\theta$ and then the bimodule structure of $\Omega^{1}(P)$ is an isomorphism. What this does is to express the cotangent bundle as associated to the principal bundle $P$. This in my view captures the essence of what a manifold is since all the ideas of charts etc are taken care of by the local triviality of the bundle, which in turn we have expressed globally by the exact sequence above.

We call a 'quantum manifold' an algebra framed in this way. A connection on $P$ then induces a covariant derivative

$$
\nabla: \Omega^{1}(M) \rightarrow \Omega^{1}(M) \underset{M}{\otimes} \Omega^{1}(M),
$$

which is just $D$ viewed under the framing isomorphism. A metric for us is an element

$$
g \in \Omega^{1}(M) \underset{M}{\otimes} \Omega^{1}(M)
$$

and corresponds to an isomorphism $\mathcal{E}^{*} \cong \mathcal{E}$. This can be expressed in turn as the idea that there is a second framing $\theta^{*}: V^{*} \rightarrow \Omega^{1}(M) P$ with $V^{*}$ in the role of $V$, what I call the 'coframing'. The corresponding metric is $\theta^{*} \otimes_{P} \theta$ applied to the canonical element of $V^{*} \otimes V$. So a 'quantum Riemannian manifold' is an algebra $M$ with a framing and coframing. 
Finally, we should have compatibility conditions between the framing and the connection. One is zero torsion and corresponds to $\bar{D} \wedge \theta=0$ where $\bar{D}$ is a suitable left-handed covariant derivative. The other is a 'metric compatibility' condition. This is the hardest to formulate and the solution proposed in [7 required a slight generalisation of Riemannian geometry itself in a manner appropriate to non-symmetric metrics (we have not demanded symmetry of $g$ above) as 'skew metric compatibility' or vanishing of 'cotorsion' $D \wedge \theta^{*}=0$.

The correspondence of this theory with conventional objects (which we need for continuity with classical geometry) takes the form

$$
g \leftrightarrow \theta, \theta^{*}, \quad R \leftrightarrow F, \quad T \leftrightarrow \bar{D} \wedge \theta, \quad \Gamma \leftrightarrow D \wedge \theta^{*}
$$

where

$$
R=((\mathrm{id} \wedge \nabla)-(\mathrm{d} \otimes \mathrm{id})) \circ \nabla, \quad \Omega^{1}(M) \rightarrow \Omega^{2}(M) \wedge \Omega^{1}(M)
$$

is the Riemann curvature,

$$
T=\mathrm{d}-\nabla \wedge, \quad T: \Omega^{1}(M) \rightarrow \Omega^{2}(M)
$$

is the torsion tensor and

$$
\Gamma=(\nabla \wedge \mathrm{id}-\mathrm{id} \wedge \nabla) g+(T \otimes \mathrm{id}) g, \quad \Gamma \in \Omega^{2}(M) \underset{M}{\otimes} \Omega^{1}(M)
$$

is our novel concept of 'cotorsion tensor'. The difference between torsion and cotorsion evidently expresses metric compatibility in a skew manner.

If one wants to be more conventional there is no problem writing axioms for symmetry of the metric in a suitable form, such as

$$
\wedge(g)=0 .
$$

Also one could be more conventional and ask for full metric compatibility under $\nabla$ in a more usual way, however this does not seem to me very natural compared to the elegance of the cotorsion approach in the frame bundle context (where we keep everything symmetric between framing and coframing) and moreover would still not achieve a unique Levi-Civita connection; that uniqueness is a very special feature of the frame bundle group classically being $O_{n}$ and cannot be expected in general.

Now we can summarise and evaluate the degree of continuity of our theory with conventional Riemannian geometry. This was a large part of the work in [7]. The summary is that the quantum Riemannian geometry that we propose reduces in the classical case to a slight generalisation of conventional geometry in which:

1. We allow any group $G$ in the 'frame bundle', hence a more general concept of a 'frame resolution' $\left(P, G, V, \theta_{\mu}^{i}\right)$ or generalised manifold. The choice of group determines the range of $\nabla$ that can be induced.

2. The generalised metric $g_{\mu \nu}=\theta_{\mu}^{* i} \theta_{\nu i}$ corresponding to a coframing $\theta_{\mu}^{* i}$ is nondegenerate but need not be symmetric.

3. The generalised Levi-Civita connection defined as having vanishing torsion and vanishing cotorsion respects the metric only in a skew sense

$$
\nabla_{\mu} g_{\nu \rho}-\nabla_{\nu} g_{\mu \rho}=0
$$

and need not be uniquely determined.

We may still impose symmetry of the metric in some natural form as above, etc., for strict continuity with classical Riemannian geometry. In this instance, however, I think it is also a good opportunity - suggested by the noncommutative Riemannian geometry - to enlarge Riemannian geometry itself a little and unify it 
with other branches of classical geometry. Thus, in the other extreme when the generalised metric is totally antisymmetric the vanishing of cotorsion minus torsion implies $\mathrm{d} g=0$ so $g$ is symplectic, as shown in [7]. This is fully in keeping with what me might expect from T-duality and other physical considerations as the first Planckian corrections to classical geometry.

We also promised a good supply of examples. Announced in Torino and to appear in [1] we have

Theorem 1 团团 All quantum groups $M$ equipped with bicovariant calculi are quantum manifolds in the above sense with framing by $H=M$ itself and $\theta$ induced by the Maurer-Cartan form in [1]]. Moreover, all standard quantum groups $\mathbb{C}_{q}[G]$ with their standard bicovariant differential calculi are quantum Riemannian manifolds in the above sense with metric induced by the braided-Killing form[18] on the braided-Lie algebra associated to the differential calculus.

From this we obtain in principle similar results for quantum homogeneous spaces including spheres, planes etc. In fact, there is a notion of comeasuring or quantum automorphism bialgebra 19 for practically any algebra $M$ and when this has an antipode (which typically requires some form of completion) one can write $M$ as a quantum homogeneous space. So almost any algebra $M$ is more or less a quantum manifold for some principal bundle (at least rather formally and so far with the universal calculus). When $M$ is equipped with more structure to define a nonuniversal differential calculus in a systematic way then this observation should extend to that level too, which would then be analogous to the idea that any classical manifold is, rather formally, a homogeneous space of diffeomorphisms modulo diffeomorphisms fixing a base point.

Also, let us mention the obvious global formulation of the Dirac operator in the frame bundle approach. Given any other vector space $W$ on which $H$ (co)acts we similarly have an associated bundle $\mathcal{S}$ say as explained above. The connection on the principal frame bundle that induced the covariant derivative $\nabla$ also induces a covariant derivative $D: \mathcal{S} \rightarrow \Omega^{1}(M) \otimes_{M} \mathcal{S}$. So the missing ingredient is just a suitable map $\gamma: \Omega^{1}(M) \rightarrow \operatorname{End}(\mathcal{S})$ which locally would be provided by $\gamma$ matrices. I am not confident about the global formulation of this map, however the paper [1] contains examples, for instance on finite groups using this bundle formulation and along these lines. i.e. the theory includes spinors in principle and has been tested on some examples.

Finally, we discuss the Ricci tensor and actions. To make the contraction for Ricci we can assume a 'lifting map' or bimodule inclusion

$$
i: \Omega^{1}(M) \underset{M}{\otimes} \Omega^{1}(M) \rightarrow \Omega^{2}(M)
$$

so as to turn $R$ as a 2-form (with values in operators on 1-forms) into an element of $\Omega^{1}(M) \otimes_{M} \Omega^{1}(M)$ (with values as before). After that we can then take a suitable trace of $i(R)$ as a left $M$-module endomorphism with values in the remaining (rightmost) copies of $\Omega^{1}(M) \otimes_{M} \Omega^{1}(M)$, which defines the Ricci tensor. Such a lift $i$ is also the ingredient needed for an interior product on the exterior algebra, so specifying this or a Hodge $*$ operation would also do the job in a natural manner. How these or $i$ are chosen depends on how the higher order differential calculus (or at least $\Omega^{2}(M)$ ) is defined but in the main cases one has some natural proposals. Assuming $i$ has been chosen we can then write down field equations via Ricci. Contracting further, we would obtain the scalar curvature. We would still need an integral for an action. Alternatively we could define the action via $\not D^{2}$ in view of the well-known Lichnerowicz formula. In the finite case we could take a trace while in general we could use the spectral action of Connes. These are some of the steps for which ambiguities exist in the frame bundle approach. I think there is no fundamental obstruction, however, to completing the programme. This is also clearly reaching the point where it should tie up with Connes' 'top down' approach but meanwhile the difference is that we are explicitly building up the different layers of infrastructure of the noncommutative geometry.

\section{Local formulae in tensor product gauge}

We are now going to give how the quantum Riemannian geometry above looks in the 'tensor product gauge' but only in outline - for details see [7] and explicitly [1] with general calculi. Also if you do not like Hopf 
algebras we give an even more explicit formulation in the next section. Thus, let $M$ be an algebra and $H$ a Hopf algebra. We take $P=M \otimes H$, which is the 'tensor product gauge' for a trivial principal bundle. We have differential calculi on $\Omega^{1}(H), \Omega^{1}(M)$ defined by subbimodules $N_{M}, N_{H}$ say. We take for $\Omega^{1}(P)$ the calculus defined by sub-bimodule

$$
N_{P}=N_{M} \otimes H \otimes H+M \otimes M \otimes N_{H}+\Omega^{1} M \otimes \Omega^{1} H
$$

one has to work to show that we fulfill the conditions for a quantum principal bundle and that in fact $P \Omega^{1}(M) P=\Omega^{1}(M) P=P \Omega^{1}(M)=\Omega^{1}(M) \otimes H$ which then allows all formulae in the global theory to be lowered to working just with $M$ and $H$. For the global picture of curvature and torsion etc., one also has to specify the global $\Omega^{2}(P)$ from $\Omega^{2}(M)$ and the $\Omega^{2}(H)$, etc. but this can be done for any reasonable $\Omega^{2}(M)$ (such as the bicovariant one if $M$ is itself a quantum group).

We then define a gauge field as any map

$$
A: \Omega_{0} \rightarrow \Omega^{1}(M)
$$

and can show that one can build up a global connection on the bundle $P$ from this data. We use Ad : $\Omega_{0} \rightarrow$ $\Omega_{0} \otimes H$ to do this. It is very important since we do not consider global gauge transformations that one does indeed get a global bundle and connection in this way 1 . Likewise other data above have local analogues as follows.

A framing amounts to a $V$-bein, i.e. a comodule $V$ (so $H$ (co)acts on it) and a linear map $e: V \rightarrow \Omega^{1}(M)$ such that it induces an isomorphism $\Omega^{1}(M) \cong M \otimes V$. If you choose a basis $\left\{e_{i}\right\}$ of $V$ then what we require is that every $\alpha \in \Omega^{1}(M)$ has the form $\alpha=\alpha^{i} e\left(e_{i}\right)$ for unique functions $\alpha^{i}$. A coframing is similarly provided by a $V$-cobein $e^{*}$ such that $\Omega^{1}(M) \cong V^{*} \otimes M$. The vanishing of torsion and cotorsion correspond to

$$
\bar{D} \wedge e=0, \quad D \wedge e^{*}=0 .
$$

Explicit formulae for $D$ on forms are part of the standard gauge bundle theory [8] and look just as in [1]. We project such equations down to $\Omega^{2}(M)$. The metric is $g=e^{*} \otimes_{M} e$ evaluated on the canonical element of $V^{*} \otimes V$.

Given a framing and $A$, the covariant derivative is

$$
\nabla \alpha=\mathrm{d} \alpha^{i} \underset{M}{\otimes} e\left(e_{i}\right)-\alpha^{i} A\left(\tilde{\pi}_{\Omega_{0}} e_{i}^{(\overline{\bar{o}})}\right) \underset{M}{\otimes} e\left(e_{i}^{(\overline{2})}\right),
$$

where the ${ }^{(\overline{0})}$ and ${ }^{(\overline{1})}$ refer to the pieces in $H \otimes V$ resulting from applying the coaction to an element of $V$. The $\tilde{\pi}_{\Omega_{0}}$ projects $H$ down to $\Omega_{0}$. The curvature corresponds to

$$
F(v)=\mathrm{d} A\left(\tilde{\pi}_{\Omega_{0}} v\right)+A\left(\tilde{\pi}_{\Omega_{0}} v_{(1)}\right) \wedge A\left(\tilde{\pi}_{\Omega_{0}} v_{(2)}\right)
$$

where the $_{(1)}$ and ${ }_{(2)}$ refer to the pieces resulting from the coproduct of $H$. We require for a well-defined map $F: \Omega_{0} \rightarrow \Omega^{2}(M)$ a regularity condition on $A$ which in this gauge appears as

$$
A\left(\tilde{\pi}_{\Omega_{0}} q_{(1)}\right) \wedge A\left(\tilde{\pi}_{\Omega_{0}} q_{(2)}\right)=0, \quad \forall q \in Q_{H},
$$

where $Q_{H} \subset H$ defines $\Omega_{0}$ for the bicovariant differential calculus on $H$. It is exactly this condition that drops out with the universal calculus on $H$, when $Q_{H}$ is zero. From the curvature we build the Riemann curvature $R$, and given a bimodule inclusion $i: \Omega^{2}(M) \rightarrow \Omega^{1}(M) \otimes_{M} \Omega^{1}(M)$ the Ricci tensor,

$$
\operatorname{Ricci}=\left\langle i(R) e\left(e_{i}\right), f^{i}\right\rangle=i\left(F_{A}\left(\tilde{\pi}_{\Omega_{0}} e_{i}^{(\bar{D})}\right)^{i j} e\left(e_{j}\right) \underset{M}{\otimes} e\left(e_{i}^{(\overline{1})}\right),\right.
$$

where $F_{A}=F_{A}^{i j} e\left(e_{i}\right) \otimes_{M} e\left(e_{j}\right)$ defines its components.

Finally, a Dirac operator is defined by a linear map $\gamma: V \rightarrow \operatorname{End}(W)$ for some other $H$-comodule $W$. It takes the form on spinors $\psi=\psi^{(1)} \otimes \psi^{(2)} \in M \otimes W$,

$$
\not D \psi=\left(\partial^{i} \otimes \gamma_{i}\right) \psi-\psi^{(1)} A^{i}\left(\tilde{\pi}_{\Omega_{0}} \psi^{(2)(\overline{0})}\right) \otimes \gamma_{i}\left(\psi^{(2)(\overline{1})}\right),
$$


where $\gamma_{i}=\gamma\left(e_{i}\right)$ and $\mathrm{d} m=\left(\partial^{i} m\right) e\left(e_{i}\right)$ defines the partial derivatives associated to the $V$-bein, and ${ }^{(\overline{0})},{ }^{(\overline{1})}$ are the pieces in $H \otimes W$ of the coaction on $W$.

These quantum group formulae may look unpalatable to anyone not happy with Hopf algebras. One may also rewrite the coaction of $H$ as an action of a dual Hopf algebra $U$ of 'enveloping algebra' type which will make them look more familiar. Moreover, see the next section.

\section{Gravity on finite sets}

We now specialise further to concrete local formulae with lots of indices, but for simplicity only in the simplest case of finite sets and framing by a classical group. It is very important conceptually and also (not so important) historically that this theory on finite sets is not something new but merely an elaboration of a more general theory essentially introduced in 1997 and that can also be specialised in other limits, e.g. noncommutative or classical geometry.

At this level, let $\Sigma$ be a finite set, $G$ a finite group, $H=\mathbb{C}[G]$ and $M=\mathbb{C}[\Sigma]$ spanned by delta-functions $\left\{\delta_{x}\right\}$ for $x \in \Sigma$. It is trivial to see (and well-known) that a general differential calculus $\Omega^{1}(M)$ corresponds to a subset

$$
E \subseteq \Sigma \times \Sigma-\text { diagonal, } \quad \Omega^{1}(M)=\left\{\delta_{x} \otimes \delta_{y} \mid(x, y) \in E\right\}
$$

where we set to zero delta-functions corresponding to the complement of $E$ and identify the remainder with their lifts as shown. If $f=\sum f_{x} \delta_{x}$ is a function with components $f_{x}$, then $\mathrm{d} f$ has components $(\mathrm{d} f)_{x, y}=f_{y}-f_{x}$ for $(x, y) \in E$.

Then a $V$-bein is a vector space on which $G$ acts by $\rho_{V}$, say, and a collection of 1 -forms

$$
E_{i}=\sum_{(x, y) \in E} E_{i, x, y} \delta_{x} \otimes \delta_{y}
$$

for each element of a basis $\left\{e_{i}\right\}$ of $V$ such that the matrices $\left\{E_{i, x, y}\right\}$ are invertible for each $x \in \Sigma$ held fixed. A necessary (and sufficient) condition for the existence of a $V$-bein is clearly that $E$ is fibered over $\Sigma$, i.e. for each $x \in \Sigma$ the set $F_{x}=\{y \mid(x, y) \in E\}$ has the same size, namely the dimension of $V$. In particular it implies that the latter is $|E| /|\Sigma|$. A natural 'local' class of $V$-beins is just given by any collection of bijections

$$
s_{x}:\{i\} \cong F_{x}, \quad E_{i, x, y}=\delta_{s_{x}(i), y},
$$

but we are not limited to such a class. Similarly a $V$-cobein is a collection of 1-forms with components $E_{x, y}^{* i}$ with respect to a dual basis $\left\{f^{i}\right\}$ and with the matrices $\left\{E_{x, y}^{* i}\right\}$ invertible for each $y \in \Sigma$ held fixed. The metric is

$$
g=\sum_{(x, y, z) \in F} g_{x, y, z} \delta_{x} \otimes \delta_{y} \otimes \delta_{z}, \quad g_{x, y, z}=E_{x, y}^{* i} E_{i, y, z},
$$

where $F$ is the subset of $(x, y, z) \in \Sigma \times \Sigma \times \Sigma$ with $(x, y) \in E$ and $(y, z) \in E$, i.e. $F$ labels the basis of $\Omega^{1}(M) \otimes_{M} \Omega^{1}(M)$.

As immediate from Woronowicz' paper [11], bicovariant calculi on $H=\mathbb{C}[G]$ are classified by nontrivial conjugacy classes or more generally (reducible ones) by Ad-stable subspaces $\mathcal{C} \subset G$ excluding the group identity $e \in G$. We denote its elements by $a, b, c$ etc., and identify the quotient with the corresponding lift so that

$$
\Omega_{0}=\left\{\delta_{a} \mid a \in \mathcal{C}\right\} .
$$

Then a connection or gauge field with values in the dual of $\Omega_{0}$ is a collection of 1-forms with components $A_{a, x, y}$. In our case $G$ acts on $V$ so that it plays the role of frame transformations in the frame bundle approach. In that case $A$ induces a covariant derivative on 1-forms

$$
(\nabla \alpha)_{x, y, z}=\left(\alpha_{y}^{i}-\alpha_{x}^{i}\right) E_{i, y, z}-\alpha_{x}^{i} A_{a, x, y} E_{j, y, z} \tau^{a j}, \quad \tau^{a}=\rho_{V}\left(a^{-1}-e\right),
$$


where $\alpha=\alpha^{i} E_{i}$ defines the component functions $\alpha^{i}$ of a 1 -form $\alpha$ in the $V$-bein basis. The $\tau^{a}$ are the matrices for the action of the 'braided-Lie algebra' dual of $\Omega_{0}$ as a subspace of the linear span $\mathbb{C} G$.

Next we specify $\Omega^{2}(M)$ by a bimodule surjection $\wedge: \Omega^{1}(M) \otimes_{M} \Omega^{1}(M) \rightarrow \Omega^{2}(M)$. We assume that this is done in a manner compatible with $\Omega^{2}(H)$ so as to fit together globally in the bundle. The surjection on any $f \in \Omega^{1}(M) \otimes \Omega^{1}(M)$ with components $f_{x, y, z}$ as above, is necessarily of the form

$$
(\wedge f)_{x, \alpha, z}=\sum_{y \in F_{x, z}} f_{x, y, z} p_{\alpha}^{y}, \quad F_{x, z}=\{y \in \Sigma \mid(x, y, z) \in F\}
$$

for a family of surjections $p: \mathbb{C} F_{x, z} \rightarrow V_{x, z}$ to some vector spaces $V_{x, z}$, obeying $p(1,1, \cdots, 1)=0$ when $(x, z) \notin E$ with $x \neq z$ (this is so that $\mathrm{d}^{2}=0$ ). Chosing a basis $\left\{e_{\alpha}\right\}$ for the latter, we write the $p$ explicitly as a family of rectangular matrices with each row summing to 0 in the stated case. When $\alpha_{x, y}, \beta_{x, y}$ are the components of 1 -forms as above then

$$
(\mathrm{d} \alpha)_{x, \alpha, z}=\sum_{y \in F_{x, z}}\left(\alpha_{x, y}+\alpha_{y, z}-\alpha_{x, z}\right) p_{\alpha}^{y}, \quad(\alpha \wedge \beta)_{x, \alpha, z}=\sum_{y \in F_{x, z}} \alpha_{x, y} \beta_{y, z} p_{\alpha}^{y} .
$$

With such an explicit description of $\Omega^{2}(M)$, a connection $A$ is regular in the tensor product gauge if

$$
\sum_{a b=q, y} A_{a, x, y} A_{b, y, z} p^{y}{ }_{\alpha}=0, \quad \forall q \notin \mathcal{C} \cup\{e\} .
$$

Its curvature is

$$
F_{a, x, \alpha, z}=\left(\mathrm{d} A_{a}\right)_{x, \alpha, z}+\sum_{c d=a, y} A_{c, x, y} A_{d, y, z} p_{\alpha}^{y}-\sum_{b, y}\left(A_{b, x, y} A_{a, y, z}+A_{a, x, y} A_{b, y, z}\right) p_{\alpha}^{y} .
$$

The actual Riemann tensor if one wants it is the 2 -form valued operator on 1 -forms,

$$
R_{x, \alpha, z^{i}}=F_{a, x, \alpha, z} \tau^{a i}{ }_{j}, \quad R \alpha=\alpha^{i} R_{i}^{j} \underset{M}{\otimes} E_{j} .
$$

Meanwhile, the zero torsion and zero cotorsion equations are vanishing of

$$
\begin{aligned}
& (\bar{D} \wedge e)_{i, x, \alpha, z}=\left(\mathrm{d} E_{i}\right)_{x, \alpha, z}+\sum_{a, j, y} A_{a, x, y} E_{j, y, z} p^{y}{ }_{\alpha} \tau^{a j}{ }_{i} \\
& \left(D \wedge e^{*}\right)_{x, \alpha, z}^{i}=\left(\mathrm{d} E^{* i}\right)_{x, \alpha, z}+\sum_{a, j, y} E_{x, y}^{* j} A_{a, y, z} p^{y}{ }_{\alpha} \tau^{a i}{ }_{j} .
\end{aligned}
$$

Also, given a 'lift' $i$, which means a collection of inclusions $i: V_{x, z} \rightarrow \mathbb{C} F_{x, z}$ or rectangular matrices $i_{y}^{\alpha}$, preferably such that $p \circ i=$ id (in which case $i \circ \wedge$ is a projection operator splitting $\Omega^{1}(M) \otimes_{M} \Omega^{1}(M)$ into something isomorphic to $\Omega^{2}(M)$ plus a complement), we have an interior product and, in particular, a Ricci tensor

$$
\operatorname{Ricci}_{x, y, z}=i\left(F_{a}\right)_{x}^{i j} E_{j, x, y} E_{k, y, z} \tau^{a k}{ }_{i}
$$

Here $i\left(F_{a}\right)_{x, w, z}$ in $\Omega^{1}(M) \otimes_{M} \Omega^{1}(M)$ is as in (37) but with $\pi^{y}{ }_{w}=p^{y}{ }_{\alpha} i^{\alpha}{ }_{w}$ in place of $p^{y}{ }_{\alpha}$ written there. These are projections if $i$ is a proper lift, but this is not strictly required. We then convert to $V$-bein components $i\left(F_{a}\right)^{i j}$ as usual. One can write this more explicitly in terms of $A, e$.

Finally, gamma-matrices are a collection of matrices $\gamma_{i}$ acting on spinors $\psi$ which are functions with values in a vector space $W$ on which $G$ acts by $\rho_{W}$, say. The associated Dirac operator is

$$
\not D=\partial^{i} \gamma_{i}-A_{a}^{i} \gamma_{i} \tau_{W}^{a}, \quad \tau_{W}^{a}=\rho_{W}\left(a^{-1}-e\right) .
$$

This is the elementary elaboration for finite sets of the general theory in preceding sections. Of course, a simple case is where $\Sigma=G$ and we use the same bicovariant calculus on both. We take $V=\Omega_{0}$ itself with 
the coadjoint action of $G$, so that the indices $i, j, k$ run over the same range $\mathcal{C}$ as the $a, b, c$. As proposed in [7] we take the $V$-bein to be the quantum group Maurer-Cartan form $e$ from [11]. There is also a natural braided-Killing form $\eta$ associated in [18] to $\Omega_{0}^{*}$ as a braided-Lie algebra, which we use to define $e^{*}=e \circ \eta$. The subset $E$ above, the exterior derivative, the components of Maurer-Cartan form and the matrices $\tau^{a}$ are explicitly,

$$
E=\left\{(x, y) \mid x^{-1} y \in \mathcal{C}\right\}, \quad \mathrm{d} f=\left(\partial^{i} f\right) E_{i}, \quad \partial^{i}=R_{i}-\mathrm{id}, \quad E_{i, x, y}=\delta_{x i, y}, \quad \tau^{a i}{ }_{j}=\delta^{i}{ }_{a}{ }^{-1} j_{a}-\delta^{i}{ }_{j},
$$

where $R_{i}$ is right-translation by $i$. Most of this is the standard starting point for any work on noncommutative differential geometry of quantum groups, translated into our above notations. Also, note that in all computations one can either work explicitly with matrices $\alpha_{x, y}$ etc. which is like 'spacetime coordinates' or work more algebraically with $V$-bein components $\alpha_{x}^{i}$ and the abstract relations in $\Omega^{1}(M), \Omega^{2}(M)($ or a mixture of the two). The conversion in the group case is particularly easy because of the translation-invariant form of $E_{i}$.

Theorem 2 [] For $G=\Sigma=S_{3}$, the permutation group on 3 elements, and $\mathcal{C}$ the maximal (order 3) conjugacy class $(i)$ the braided Killing form is $\eta^{i j}=3 \delta^{i j}$ and defines the metric $g_{x, y, z}=3 \delta_{x^{-1} y, y^{-1} z}$, (ii) there is a unique torsion-free and cotorsion-free regular or 'generalised Levi-Civita' connection for this metric, given by $A_{a, x, y}=\delta_{x a, y}-\frac{1}{3}$.

We also look in [1] at two natural 'lifts' in the finite group case. One is the Woronowicz lift whereby $i=\mathrm{id}-\Psi$. The kernel of this map is precisely the elements in $\Omega^{1}(M) \otimes_{M} \Omega^{1}(M)$ set to zero by $\wedge$, so we can identify $\Omega^{2}(M)$ with its image. Since $\Psi^{2} \neq$ id for a nonAbelian $G$, it is not a precise lift in the sense of $i \circ \wedge$ a projection. Another is an actual lift constructed in [1] giving a projection operator for all finite groups. In either case one finds [1]

$$
\operatorname{Ricci}_{x, y, z}=-\mu\left(g_{x, y, z}-1\right)
$$

where $\mu$ is a positive constant. The further contraction, the scalar curvature, is also constant and negative. Note that this is like a sphere in our preferred conventions for Ricci (where we contracted what would classically be the first and third indices of $R$, i.e. minus the usual conventions).

Finally, there are natural $2 \times 2$ equivariant $\gamma$-matrices obeying

$$
\left\{\gamma_{i}, \gamma_{j}\right\}+\frac{2}{3}\left(\gamma_{i}+\gamma_{j}\right)=\frac{1}{3}\left(\delta_{i j}-1\right)
$$

and the corresponding Dirac operator turns out to be[1]

$$
\not D=\partial^{i} \gamma_{i}-1
$$

This is just the beginning (the natural Riemannian structure on $S_{3}$ ). Clearly we have the machinery above to consider the moduli of all $\left(A, e, e^{*}\right)$ and solve field equations or Legendre transform action functionals with respect to them, i.e. classical and quantum gravity. If one wants a more conventional moduli space for the metric then one can fix the relationship $e^{*}=e \circ \eta$ as above and only vary $(A, e)$. We will consider the moduli space elsewhere.

\section{Quantum measurement and algebra bundles}

As mentioned in the introduction, there is one further generalisation beyond the case of quantum group (or classical group) framing that is needed for a fully comprehensive theory of Riemannian geometry that would apply in principle to real-world quantum systems. That is to the level of only coalgebras in place of $H$ above, or to algebras in place of its 'enveloping type' dual Hopf algebra $A$. Such a theory more or less exists as the 
theory of (co)algebra bundles in [20] at the gauge theory level and [9] at the frame bundle level. The basic idea is the following. Given a classical or quantum bundle as above, one can make a cross product

$$
X=P \rtimes A
$$

by the action of $A$ on $P$. Like all cross products this factorises into $P$ and $A$ as subalgebras. The generalisation is to consider more general algebras $X$ that factorise $X=P A$ as vector spaces, where $P, A$ are subalgebras. In addition we require a map $e: A \rightarrow P$ with certain properties corresponding to the exact sequence that defined a global bundle in Section 3. It turns out then that most of the global gauge theory and Riemannian geometry above, i.e. connections as projections of $\Omega^{1}(P)$, associated bundles, frames, etc. then goes through even though $A$ is not a Hopf algebra at all.

To give some idea of the constructions in [20] [9], first note that when $X$ factorises there is an induced 'reordering map' $\Psi: A \otimes P \rightarrow P \otimes A$ called the entwining or factorisation structure, and when $e$ is given obeying

$$
e(a b)=\cdot(\mathrm{id} \otimes e) \Psi(a \otimes e(b)), \quad e(1)=1,
$$

one has an action

$$
a \triangleright p=\cdot(\operatorname{id} \otimes e) \Psi(a \otimes p)
$$

of $A$ on $P$. This is such that $P$ acting on itself and this action of $A$ fit together to an action of $X$ on $P$. Next, we define $M$ as the 'fixed subalgebra' by

$$
M=\{m \in P \mid a \triangleright m=e(a) m, \quad \forall a \in A\}=\{m \in P \mid a \triangleright(p m)=(a \triangleright p) m, \quad \forall a \in A, p \in P\} .
$$

We can then view the action of $A$ more precisely as the coaction of a coalgebra $C$ dual to $A$ and define ver : $\Omega^{1} P \rightarrow P \otimes \operatorname{ker} \epsilon$ as in the exact sequence before, where ker $\epsilon$ are the elements of $C$ that vanish on 1. This is for the universal differential calculus. The theory with general differential calculus is not fully elaborated but exists in principle; we need a quotient $\Omega_{0}$ of ker $\epsilon$ with suitable properties and the similar exact sequence. Not that these conditions relate to the differential geometric structure of the gauge group and are therefore important for the global bundle structure but not at the local level. For example, for finite sets above we needed for most of the theory only that $\Omega_{0}$ was some chosen quotient and none of its properties (until we took $G$ also as spacetime). Also Ricci and the Dirac operator are not yet elaborated in this context, but are not a problem in principle.

The first point of interest for conventional quantum theory is that the converse is also true: if $A$ is an algebra acting on the vector space of some algebra $P$, define $M$ by the second version in (50), let $e(a)=a \triangleright 1$ and suppose that we have the exact sequence or 'local triviality' condition. Then one gets a bundle and a map $\Psi$ leading to a generalised braided tensor product algebra

$$
X=P \underline{\otimes}_{\Psi} A
$$

factorising into $P, A$ and still represented on $P$ (with $P$ acting by multiplication). Here the product $(q \otimes a)(p \otimes b)$ is to use $\Psi$ to take $a$ past $p$ and then multiply in $A$ and in $P$.

We now outline how this mathematics could form the basis of a quantum theory of measurement. Usually we have some fairly naive 'postulates' that a measurement is deemed to have taken place when a wave-function is projected into an eigenstate of some chosen operators. The problem is that from a larger point of view the measurement itself should be a process in a larger quantum system and only appearing like the above in some idealisation. In the larger system we would need to identify the algebraic structures which would become the macroscopic parts of the measuring apparatus and the remainder the quantum part in that idealisation. Such identifications or correspondences is exactly the task of quantum geometry. Now in quantum mechanics this geometrical structure is usually buried in the Hilbert space, which is typically of the form $L^{2}(C)$ for some 'configuration space' $C$. It is not just some abstract Hilbert space (these are all isomorphic) because 
the structure of $C$ goes into the construction of the Hamiltonian and other correspondences between the classical and quantum theory. Forgetting about the $L^{2}$ completion, we are therefore in the situation where our quantum system is an algebra $A$ acting on the vector space of some other algebra $P$. The algebraic structure of $P$ carries the geometric structure of $C$. This exactly the situation above, so we have as an immediate application of 20] [9],

Corollary 3 (i) For any quantum system A acting on (the Hilbert space completion of) an algebra of wavefunctions $P$, we have a subalgebra $M \subseteq P$ defined by (50), the 'superselection algebra' of the quantum system. We say that the system is reduced if $M$ is trivial. (ii) If the exact sequence condition holds so that we have a bundle (we say that the quantum system is Galois) then we have an extended quantum system $P \underline{\otimes}_{\Psi} A$ containing both the quantum system $A$ and the algebra $P$ of functions on the configuration space, represented together on $P$.

In the Galois case the bigger algebra contains both what was to be measured (the quantum system) and the classical system $P$ of functions on the configuration space wherin measurements lie (in the sense that the norm of the value at $c$ is the probability density to measure $c \in C$ ). As well as a method of constructing new quantum systems from old (on the same Hilbert space), this is also a method of constructing quantum systems in the first place from a representation of one part $A$ that we would like to include in another part $P$; even if both parts are classical the result $X$ can be quantum. Actually, this is a very typical construction for quantum systems, where we typically seek to represent momentum modes on the configuration space and then throw in the configuration space coordinates acting by multiplication as well (the extended system). The theory above gives a mechanism for such a construction based just on the act of representing one part $A$ in another part $P$ subject to a nondegeneracy (Galois) condition. A concrete example is a dynamical system where the algebra $A$ generated by a group $G$ acts on the algebra of functions $P$ on a space $C$. The space $C$ foliates into orbits and $M$ is the algebra of functions on the space $C / G$ of orbits. The system is reduced if there is just one orbit. Otherwise $M$ describes the superselection sectors of the theory. Here $X=P \rtimes A$ is what is usually called the quantisation of the dynamical system, represented on $P$. The geometric picture here is also clear; $C$ in nice cases is a bundle over $C / G$. This shows how the above theory is already a useful model of conventional quantum mechanics. We can ask which familiar systems are reduced? Which are Galois? Moreover, in the latter case we have in principle all the machinery of bundles, etc. to apply quite generally, with $M$ in the role of base manifold even for quantum systems not obviously of geometric origin.

On the other hand there is no reason why $P$ (or $A$ or $M$ ) should be commutative, i.e. all concepts in the formulation above work when the system is already noncommutative or quantum. We therefore have the possibility of quantising a system with 'wave-functions' actually operators in some already quantised system $P$. Thus we could ask if one part $A$ of a quantum system is represented on another part $P$ and build the extended system. This is a necessary (though not sufficient) part of the solution of the measurement problem because it begins to formulate procedures in a manner applicable before making any idealisations to the special form of a classical configuration space, i.e. measuring quantum theory within quantum theory or an intrinsic theory of measurement involving some parts 'measuring' other parts. Moreover, we still have the geometric picture but now as quantum bundles, quantum Riemannian geometry etc. for such systems. Finally, we also have the first part of the theory above which goes in the other direction: a theory of factorisations of a bigger system $X$ into a part that we would like to be a smaller quantum system $A$ and a part that we would like to be (only approximately if noncommutative) a classical or macroscopic system $P$.

Putting these ideas together we arrive at a relative theory of quantum measurement which we propose along the following lines.

1. A quantum system $A$ means a $*$-algebra.

2. A relative 'wave function' state is an element $p$ of another possibly quantum system $P$ on which $A$ is represented as a vector space. We call $1 \in P$ the 'character state'. 
3. We call $\langle a\rangle_{p}=p^{*}(a \triangleright p)$ the 'relative expectation value of $a$ in state $p$ '. More generally, a relative expectation is any positive map $\phi: A \rightarrow P$.

4. If $P$ is itself represented in some other system $Q$ then $\left\langle\langle a\rangle_{p}\right\rangle_{q}$ is the expectation of the above expectation in state $q$, etc.

5. When the representation of $A$ in $P$ is Galois, we may view them as subalgebras of a bigger quantum system $X=P A$.

6. Conversely, if a quantum system $X$ factorises as $X=P A$ and a relative expectation $e: A \rightarrow P$ obeying (48) is specified then $A$ is represented in $P$ and $e(a)=\langle a\rangle_{1}$.

This relative theory unifies both the Schroedinger and the $C^{*}$-algebra type attitudes towards quantum mechanics. Thus, in one extreme, take $P=\mathbb{C}$. Then a relative expectation is just a positive linear map $\phi: A \rightarrow \mathbb{C}$, i.e. states from a $C^{*}$-algebra type point of view. A representation in $\mathbb{C}$ is determined by a character $e: A \rightarrow C$ and $\langle a\rangle_{1}=e(a)$. We view $\mathbb{C}$ as the 'end of the line' in that we do not expect to represent this elsewhere, and can speak (glibly) about actual expectations in this case. However, our view in general is a Bayesian one in which all probability is relative. In another special case take $P$ functions on a classical space $C$. Then wave functions are usual wave functions. $\langle a\rangle_{p}$ is also a wave-function, the expectation density. A further representation of the classical system $P$ in $Q=\mathbb{C}$ is a point $c \in C$ and $\langle p\rangle_{1}=p(c)$. More generally, an expectation state $\phi: P \rightarrow \mathbb{C}$ is a convex combination of points in $C$ or a probability measure, more precisely. Then composing $\langle a\rangle_{p}$ with this gives the actual expectation value of $a$ in the usual sense. For example, if we chose $\phi$ to be the uniform probability distribution then $\phi\left(\langle a\rangle_{p}\right)$ is the usual quantum mechanical expectation. It corresponds to the 'all other things being equal' assumption in the final step to get from a relative probability to a so-called absolute one. We would also expect a relative GNS-type construction based on quotienting the regular representation of $A$ relative to a general state $A \rightarrow P$, etc. This should go hand in hand with some notion of relative completions needed to make the above precise but without assuming that every algebra is normed (or a $C^{*}$ algebra) to begin with (this is optional but would be in keeping with the relative philosophy).

While not a full theory of quantum measurement, we have indicated here the start of a general framework. Moreover, this framework has all the tools of noncommutative Riemannian geometry, gravity etc. as explained above, at our disposal. Putting these together one would expect a full resolution of the link between measurement, entropy and gravity. Also note that, while not Galois, important algebra factorisations arose in the theory of Hopf algebra factorisations. In those models one has dual Hopf algebras related to Hopf algebra duality and observable state duality [2]. This too should be generalised and resolved using the above formulation.

\section{Acknowledgements}

It is a pleasure to thank the organisers of the Euroconference for a thoroughly enjoyable and stimulating event.

\section{References}

[1] S. Majid. Riemannian geometry of quantum groups and finite groups with nonuniversal differentials. In preparation.

[2] S. Majid. Hopf algebras for physics at the Planck scale. J. Classical and Quantum Gravity, 5:1587-1606, 1988.

[3] S. Majid. Quantum random walks and time reversal. Int. J. Mod. Phys., 8:4521-4545, 1993.

[4] S. Majid. On q-regularization. Int. J. Mod. Phys. A, 5(24):4689-4696, 1990. 
[5] G. Amelino-Camelia and S. Majid. Waves on noncommutative spacetime and gamma-ray bursts. Int. J. Mod. Phys. A, (in press).

[6] R. Oeckl. Braided quantum field theory. Preprint, 1999.

[7] S. Majid. Quantum and braided group Riemannian geometry. J. Geom. Phys., 30:113-146, 1999.

[8] T. Brzeziński and S. Majid. Quantum group gauge theory on quantum spaces. Commun. Math. Phys., 157:591-638, 1993. Erratum 167:235, 1995.

[9] T. Brzeziński and S. Majid. Quantum geometry of algebra factorisations and coalgebra bundles. Commun. Math. Phys., (in press).

[10] T. Brzeziński and S. Majid. Quantum differentials and the q-monopole revisited. Acta Appl. Math., 54:185-232, 1998.

[11] S.L. Woronowicz. Differential calculus on compact matrix pseudogroups (quantum groups). Commun. Math. Phys., 122:125-170, 1989.

[12] S. Majid. Classification of bicovariant differential calculi. J. Geom. Phys., 25:119-140, 1998.

[13] E. Beggs and S. Majid. Quasitriangular and differential structures on bicrossproduct Hopf algebras. J. Algebra, 219:682-727, 1999.

[14] S. Majid and R. Oeckl. Twisting of quantum differentials and the Planck scale Hopf algebra. Commun. Math. Phys., 205:617-655, 1999.

[15] A. Connes. Noncommutative Geometry. Academic Press, 1994.

[16] S. Majid. Quantum geometry of field extensions. J. Math. Phys., 40:2311-2323, 1999.

[17] S. Majid. Foundations of Quantum Group Theory. Cambridge University Press, 1995.

[18] S. Majid. Quantum and braided Lie algebras. J. Geom. Phys., 13:307-356, 1994.

[19] S. Majid. Quantum and braided diffeomorphism groups. J. Geom. Phys., 28:94-128, 1998.

[20] T. Brzezinski and S. Majid. Coalgebra bundles. Commun. Math. Phys., 191, 1998. 467-492. 